\title{
Asteroseismic study of solar-like stars: A method of estimating stellar age
}

\author{
Y. K. Tang ${ }^{1,3}$ S. L. Bi ${ }^{2,1}$ and N. Gai ${ }^{1,3}$ \\ ${ }^{1}$ National Astronomical Observatories/Yunnan Observatory, Chinese Academy of Sciences, \\ Kunming 650011, P. R. China \\ email: bisl@bnu.edu.cn; tangyanke@ynao.ac.cn \\ ${ }^{2}$ Department of Astronomy Beijing Normal University, Beijing 100875, P. R. China \\ ${ }^{3}$ Graduate School of the Chinese Academy of Sciences, Beijing 100039, P. R. China
}

\begin{abstract}
Asteroseismology, as a tool to use the indirect information contained in stellar oscillations to probe the stellar interiors, is an active field of research presently. Stellar age, as a fundamental property of star apart from its mass, is most difficult to estimate. In addition, the estimating of stellar age can provide the chance to study the time evolution of astronomical phenomena. In our poster, we summarize our previous work and further present a method to determine age of low-mass main-sequence star.
\end{abstract}

Keywords. Stars: evolution, stars: oscillations

\section{Introduction}

Due to the frequencies of these oscillations depend on density, temperature, gas motion, and other properties of the stellar interior, it can take the window to "see" the interior of stars and help us to know the stellar internal structure and understand the stellar evolution. With the advance of observational technique, several stars have been detected the solar-like oscillations. Using the latest asteroseismic data, we reconstruct the model of $\alpha$ Cen B and 70 Ophiuchi A (Tang et al. 2008a, 2008b). In additional, Bi et al. (2008) have performed preliminary seismological analysis of two MOST targets.

\section{Using asteroseismic diagram to estimate stellar age}

Following the asymptotic formula for the frequency $\nu_{n, l}$ of a stellar $p$-mode of order $n$ and degree $l$ was given by Tassoul(1980):

$$
\nu_{n, l} \simeq\left(n+\frac{l}{2}+\epsilon\right) \nu_{0}-[A l(l+1)-B] \nu_{0}^{2} \nu_{n, l}^{-1},
$$

where, $\nu_{0}$ and $A$ are related to the run of sound speed. Based on some quantities as diagnostic purpose to probe the stellar interior proposed by some authors (ChristensenDalsgaard 1988; Gough 2003; et al.), like $\delta \nu_{n, l}=\nu_{n, l}-\nu_{n-1, l+2}$, we definite another quantity (Tang et al. 2008)

$$
r_{01}=\frac{\left\langle\delta \nu_{0,2}\right\rangle}{\left\langle\delta \nu_{1,3}\right\rangle}
$$

The $r_{01}$ comes from the perturbation to the gravitational potential, neglected in equation (2.1), which affects modes of the lowest degrees most strongly and which probably increases with evolution due to the increasing central density for modes of the lowest degrees which penetrate most deeply and hence affect $\delta \nu_{0,2}$ more than $\delta \nu_{1,3}$, leading to the dependence of $r_{01}$ on age. We compute some models with initial heavy metal 

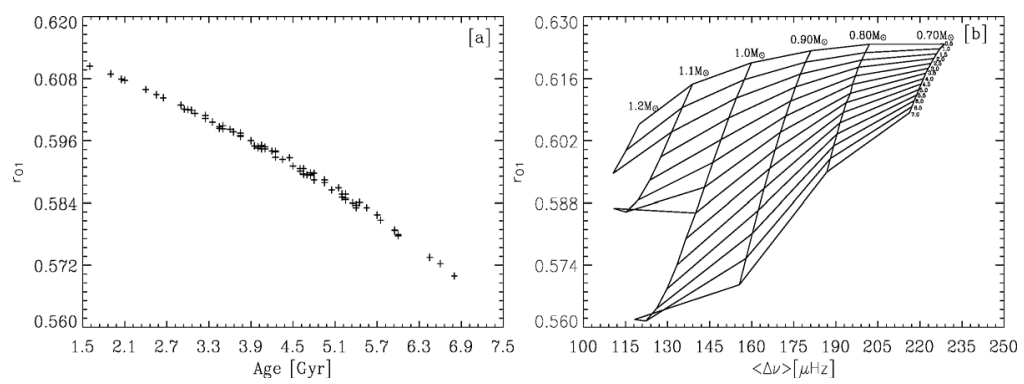

Figure 1. [a]: The ratio of small separations adjacent in $l$ vs. age for each of 129 stellar models described in Tang et al. (2008a). [b]: $\left(\langle\Delta \nu\rangle, r_{01}\right)$ diagram for stellar models. The vertical lines are evolutionary tracks, labeled by the mass in the top, whereas the transverse lines are isopleths with constant age, labeled by the age from 0.5 Gyr to 7.0 Gyr increasing with 0.5 (unit is Gyr).

abundance $Z_{i}=0.02$, initial helium abundance $Y_{i}=0.28$ and mixing-length parameter $\alpha=1.7$ using the Yale stellar evolution code (YREC; Guenther et al. 1992) and analyze the pulsation of low degree $p$-modes $(l=0-3)$ for selected models in each given mass is implemented using the Guenther's pulsation code under the adiabatic approximation (Guenther 1994). Considering the $r_{01}$ is tightly correlated with age, we construct another asteroseismic diagram shown in Fig. 1b, based on the values obtained from the above computation. Interestingly, the age of star can be marked in this asteroseismic diagram Fig. 1b. It is convenient to obtain the stellar important parameters: the mass and the age.

\section{Discussion}

1. The $\left(\langle\Delta \nu\rangle, r_{01}\right)$ diagram as a new asteroseismic diagnostic tool can estimate the mass and the age of solar-like stars. The virtue is that the age of stars can be marked in the diagram, so we can obtain the mass and age directly.

2. We will discuss the effects of the assumed initial abundance of helium and the mixing length parameter on the asteroseismic diagram in future work.

\section{Acknowledgements}

This work was supported by The Ministry of Science and Technology of the People's republic of China through grant 2007CB815406, and by NSFC grants 10173021, 10433030, 10773003, and 10778601.

\section{References}

Bi, S. L., Basu, Sarbani \& Li, L. H. 2008, ApJ 673, 1093

Christensen-Dalsgaard, J. 1988, In Proc. IAU Symposium No 123, Advances in helio- and asteroseismology, p. 295-298, eds Christensen-Dalsgaard, J. \& Frandsen, S., Reidel, Dordrecht

Gough, D. O. 2003, AP\&SS, 284, 165

Guenther, D. B., et al. 1992, ApJ 387, 372

Guenther, D. B. 1994, ApJ 422, 400

Tang, Y. K., Bi, S. L., \& Gai, N. 2008a, New Astronomy 13, 541

Tang, Y. K., Bi, S. L., \& Gai, N. 2008b, ChJAA, in press

Tassoul, M. 1980, ApJS 43, 469 